Urologe $2009 \cdot 48: 227$

DOI 10.1007/s00120-008-1916-x

Online publiziert: 20. März 2009

๑) Springer Medizin Verlag 2009

\author{
M. Goepel \\ Klinik für Urologie und Kinderurologie, Klinikum Niederberg, Velbert
}

\title{
Medikamentöse Therapie bei Blasenfunktionsstörungen
}

Tim Schneider aus Mülheim/Ruhr geht

Das Leitthema dieses Heftes befasst sich mit dem täglichen Brot des Urologen in Klinik und Praxis, nämlich der medikamentösen Therapie von Blasenfunktionsstörungen und bei Harninkontinenz. Matthias Oelke, MHH Hannover, geht der Frage nach, bei welcher Form der Belastungsinkontinenz eine (dauerhafte) medikamentöse Therapie möglich und sinnvoll ist. Hierbei ist immer auch der minimal-invasive operative Eingriff als Alternative ins Kalkül zu ziehen. Wie wir alle aus der täglichen Praxis wissen, ist Duloxetin inzwischen auch eine Therapiealternative beim belastungsinkontinenten männlichen Patienten, ohne dass hierzu inzwischen eine Zulassung zur Therapie vorläge. Der Hersteller ist aufgefordert, diese Studie zu initiieren.

Ein weiteres äußerst erfolgreiches Therapieschema bei Blasenfunktionsstörungen ist die Detrusorinfiltration mit Botulinumtoxin A. Eine Reihe von Arbeitsgruppen hat dieses Verfahren in der etablierten Indikation der therapierefraktären neurogenen Dysfunktion prospektiv getestet und nicht zuletzt aufgrund der sehr geringen Nebenwirkungen die Indikation auch auf die therapierefraktäre nicht-neurogene Blasendysfunktion ausgeweitet. Hier liegt ebenfalls keine Zulassung vor und die Therapie muss im Einzelfallverfahren mit den Kassen abgeklärt werden. Fragen nach der besten Dosis oder dem besten Injektionsort sind bei beiden Indikationen noch unklar und bedürfen weiterer Studien. Trotzdem sollte die Zulassung für die nicht-neurogene therapieresistente überaktive Blase im Interesse der Patienten vorangetrieben werden. der Frage nach, ob und wenn ja - worin sich die verschiedenen medikamentösen Alternativen bei der Überaktiven Blase unterscheiden. Der Stellenwert der neueren Substanzen Darifenacin, Solifenacin und Fesoterotin wird dargelegt und die Differentialindikation OAB wet und OAB dry differenziert.

Klaus Höfner, Oberhausen, Vorsitzender des Arbeitskreises BPH der Akademie der Deutschen Urologen, versucht die Frage zu klären, ob und wann medikamentöse Kombinationstherapien bei Symptomen des unteren Harntraktes (LUTS) verursacht durch BPH Sinn machen. Die sich ergebenden additiven Therapieeffekte werden dabei auch hinsichtlich ihrer ökonomischen Implikationen kritisch beleuchtet. Bisher ist es nicht gelungen, die definitive Therapie der BPH/ BPO medikamentös sicher und lebenslang zu verhindern.

Auch Richard Berges aus Köln, Mitglied des gleichen Arbeitskreises, beleuchtet die Frage, wann medikamentöse Therapien bei Funktionsstörungen des unteren Harntraktes indiziert sind und wann die definitive und operative Therapie ins Auge zu fassen ist.

Martin Michel, Amsterdam, der die urologisch-pharmakologische Forschung auf dem Gebiet des unteren Harntraktes seit einem Jahrzehnt wesentlich stimuliert, hat das oft vernachlässigte Gebiet der Arzneiinteraktionen in der Urologie bearbeitet. Dieses Kapitel sei jedem Kollegen ans Herz gelegt, der Pharmakotherapie am Harntrakt betreibt oder z.B. von neurologischen Kollegen wegen Miktionsstörungen bei Parkinson-Patienten um Hilfe gebeten wird.
Insgesamt halte ich dieses Leitthema für äußerst wichtig, praxisrelevant und damit lesenswert.

Mit den besten Empfehlungen

Ihr

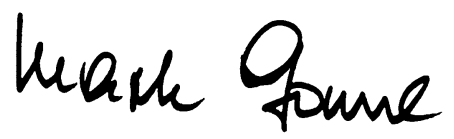

Prof. Dr. Mark Goepel

Federf. Schriftleiter „Der Urologe“

\section{Korrespondenzadresse}

Prof. Dr. M. Goepel

Klinik für Urologie und Kinderurologie,

Klinikum Niederberg,

Robert-Koch-Str. 2, 42549 Velbert

goepel@klinikum-niederberg.de 